\title{
Cardiovascular Disease-Related Lifestyle Factors among People with Type 2 Diabetes in Pakistan: A Multicentre Study for the Prevalence, Clustering, and Associated Sociodemographic Determinants
}

\author{
Ali Khan Khuwaja, ${ }^{1}$ Saima Lalani, ${ }^{2}$ Iqbal Syed Azam, ${ }^{3}$ Badar Sabir Ali, ${ }^{4}$ \\ Abdual Jabbar, ${ }^{5}$ and Raheem Dhanani6,7 \\ ${ }^{1}$ Department of Family Medicine and Community Health Sciences, Aga Khan University, Karachi 74880, Pakistan \\ ${ }^{2}$ Medical College, Aga Khan University, Karachi 74880, Pakistan \\ ${ }^{3}$ Statistical Consulting Clinics, Department of Community Health Sciences, Aga Khan University, Karachi 74880, Pakistan \\ ${ }^{4}$ Pakistan Association of Mental Health, Karachi 74880, Pakistan \\ ${ }^{5}$ Section of Diabetes and Endocrinology, Aga Khan University, Karachi 74880, Pakistan \\ ${ }^{6}$ Department of Family Medicine, Aga Khan University, Dar es Salaam, Tanzania \\ ${ }^{7}$ Department of Family Medicine, McGill University, Montreal, QC, Canada H3A 3R1
}

Correspondence should be addressed to Ali Khan Khuwaja, ali.khuwaja@aku.edu

Received 15 September 2010; Revised 1 February 2011; Accepted 9 June 2011

Academic Editor: Christina Chrysohoou

Copyright ( 2011 Ali Khan Khuwaja et al. This is an open access article distributed under the Creative Commons Attribution License, which permits unrestricted use, distribution, and reproduction in any medium, provided the original work is properly cited.

\begin{abstract}
Background. We evaluated the prevalence and clustering pattern of cardiovascular disease (CVD) related lifestyle factors and their association with CVD among patients with type 2 diabetes. We also examined the association of these factors with various sociodemographic characteristics. Methods. A total of 1000 patients with type 2 diabetes were interviewed in a cross-sectional, multicenter study in out-patient clinics in Karachi, Pakistan. Results. In this study 30.3\% study participants had CVD. Majority of the patients were physically inactive and had adverse psychosocial factors. Forty percent of the study participants were exposed to passive smoking while $12.7 \%$ were current smokers. Only $8.8 \%$ of study subjects had none of the studied lifestyle factor, $27.5 \%$ had one, while $63.7 \%$ had two or three factors. CVDs were independently associated with physical inactivity, adverse psychosocial factors, passive smoking and clustering of two or three lifestyle factors. Physical inactivity was more prevalent among females and patients with no/less education. Proportion of adverse psychosocial factors were higher among females, elders and patients with no/less education. Clustering of these lifestyle factors was significantly higher among females, elderly and no/less educated patients. Conclusion. These results suggest the need of comprehensive and integrated interventions to reduce the prevalence of lifestyle factors.
\end{abstract}

\section{Introduction}

Cardiovascular disease (CVD) is known to be the leading cause of mortality worldwide resulting in 17.1 million deaths, with 13 million deaths attributed to coronary heart disease and stroke alone; of these, more than $80 \%$ of deaths has occurred in low- to middle-income countries [1]. It is projected that by the year 2030, CVD-related mortality will rise up to 25 million, mainly from heart disease and stroke
[1]. Similarly, global statistics on diabetes are also alarming, as the disease is rapidly increasing worldwide and future projections of its burden are reported to rise particularly in Pakistan and other developing countries $[2,3]$.

It is evident that diabetes is an independent risk factor for CVD, and people with diabetes are three to four times more likely to develop CVD $[4,5]$. The development of CVD in diabetic patients is a cause of concern, as it is a major reason for hospitalization, premature morbidity, disability, 
and mortality $[6,7]$ which lead to an increased cost of care. It is reported that over $70 \%$ of the cost attributed to diabetes care is associated with its cardiovascular complications [6].

Lifestyle behaviors such as lack of exercise, psychosocial factors, and exposure to smoking further increase the risk of developing CVD [8-12] and are also highly prevalent in less developed countries $[13,14]$. On the other hand, prevention and modification of these unhealthy behaviors can lead to reduction in CVD and resultant mortality $[13,15]$. Literature reveals that at least $80 \%$ of CVD (coronary heart disease and cerebrovascular diseases) could potentially be avoided by adopting healthy lifestyle $[1,15]$.

The clustering of such unhealthy lifestyle practices has very important implications for both public health practitioners as well as for clinicians. It is well known that the risk of developing CVD multiplies many folds when related lifestyle risk factors coexist as compared to their individual risk [13]. Numerous studies have identified the clustering pattern of CVD-related factors among various groups of population [16, 17]. A study from Japan reported the clustering of cardiovascular risk factors, but the study mainly focused on biological risk factors such as glucose intolerance, dyslipidemia, and hyperuricemia [17]. Recently, Khuwaja and Kadir reported the clustering of lifestyle risk factors in Pakistan [13], but the study participants did not include a high-risk population for CVD such as patients having diabetes. We therefore conducted this study among diabetic patients to assess the prevalence and clustering pattern of CVD-related lifestyle risk factors and their associations with CVD. We also aimed to identify the sociodemographic characteristics associated with these lifestyle risk factors.

\section{Materials and Methods}

This multicentre cross-sectional study was conducted in four outpatient clinics in Karachi, the largest city and economic hub of Pakistan with a population of over 16 million, which represent all ethnicities and socioeconomically diverse populations in the country. An attempt to capture a wide spectrum of clinical and socioeconomic factors was made by enrolling study participants from four different clinics including family medicine, internal medicine, specific diabetes care, and endocrinology clinics representing both public and private sectors.

All patients having type 2 diabetes visiting their respective clinics for follow-up visits were consecutively recruited till the final sample size was achieved. We considered patients having type 2 diabetes who were labeled as "type 2 diabetic patients" by their treating physician and recorded in their medical files. However, patients suffering from type 1 diabetes or women with gestational diabetes were excluded. In this study, we were not expecting any adverse event to study participants; even so, prior to enroll in the study, consent was obtained from all the study participants. Participants were assured about the confidentiality and anonymity of their information, and all efforts were made to ensure privacy. After reviewing the study protocol and questionnaire, permission was granted from all the concerned clinics to conduct this study in their clinics.

Face-to-face interviews were conducted to get the required information, and each interview took about 20 minutes. Pretested structured questionnaire was used to obtain information about sociodemographic (gender, age, and education level) and lifestyle risk factors (level of physical activity, psychosocial history, smoking status, and exposure to passive smoking). Physical activity was assessed by using International Physical Activity Questionnaire (IPAQ) [18]. Having either anxiety and/or depression was used as proxy indicator for adverse psychosocial factors. The questionnaire used to assess the presence of anxiety and depression was Hospital Anxiety and Depression Scale (HADS) [19]. This tool is validated in national language of Pakistan (Urdu) [20] and was used extensively to study anxiety and depression among various outpatient as well as inpatient settings. One of its main features is that the items, which could relegate to problems such as insomnia, unemployment, eating disorders, headache, and fatigue, have been excluded, in order to avoid false positive cases among persons with somatic diseases. The HADS-D (Depression) covers mostly anhedonia and loss of interest, which form the core of depression symptoms, whereas HADS-A (Anxiety) covers mainly the fields of tension and worry. The HADS questionnaire consists of 14 items (sentences-questions) with answers in four grades on a Likert scale. It has seven questions regarding anxiety and seven for depression. Maximum sum was 21 for each scale. HADS uses $>8$ as a "cutoff" score. A score up to 8 indicates that the individual is free of symptoms, and a score beyond 8 defined that the symptomatology of anxiety or depression is present.

The variables which included blood pressure, glycemic levels, lipid profile, height, and weight were noted/verified from patients' medical records. Body mass index (BMI) was calculated as weight in kilograms divided by height in meter squares. Study participants were classified as obese if their BMI $>25 \mathrm{~kg} / \mathrm{m}^{2}$. Hypercholesterolaemia was defined according to ATP III criteria ( $\geq 200 \mathrm{mg} / \mathrm{dL}$ ). Individuals were classified as hypertensive if they were previously diagnosed and currently on antihypertensive medication. However, participants in whom elevated levels of blood pressure were discovered for first time at the day of interview (indexed visit) were not included in hypertensive category, as it is recommended to have at least two elevated blood pressure readings at two different times. Cardiovascular disease (coronary artery disease and/or stroke) was considered to exist if there was a history of coronary heart disease (angina, myocardial infarction) verified through medical records of a prior episode and confirmed by work-up including electrocardiography, echocardiography, and exercise treadmill test, while having stroke was solely labeled on the basis of patient's history along with file note verification of treating physician. CVD-related lifestyle factors such as physical inactivity were defined as not doing moderate to vigorous activity for 30 minutes or more, at least 4 days in a week, and participants falling in this group were categorized as "physically inactive." Patients having anxiety and or depression were categorized positive for "adverse psychosocial factor." Persons smoking 
any number of cigarettes per day were classified as "current smokers." Passive smoker for this study was defined as any person who has been exposed to second-hand smoke for at least 30 minutes in a day, for at least 5 days of the week either at home and or at work place, for last six months, and more.

To analyze the defined objectives of this study, the initial required sample size was 895 study participants. However, we approached 1000 consecutively eligible type 2 diabetic patients from the four clinics to participate in the study. In total, 87 patients refused to participate in the study, while the required information was incomplete and missing for 26 patients. Therefore, the final analysis was performed on 887 study participants. All the data was collected within eightmonth duration. All the data was collected, cleaned, and validated by medical graduates specifically trained for this task.

The data was analyzed using the Statistical Package for Social Sciences (SPSS) version 19. Mean and standard deviations (SD) for continuous variables and percentages for categorical variables and lifestyle factors were calculated separately. Clustering of lifestyle factors was studied as none, one, and two or three. In model I, multivariable analysis using multiple logistic regressions was carried out to evaluate the association of combined effect of lifestyle factors with CVD among patients with type 2 diabetes, while in model II, the association of clustering pattern of lifestyle factors with CVD was assessed. Age, sex, educational status, and body mass index are well known confounders for lifestyle factors and CVD [7, 13]; we therefore adjusted these variables in both models to evaluate the independent association of CVD with lifestyle risk factors and their clustering pattern. Crosstabulation and chi-square test of significance was applied to identify the association of lifestyle factors and clustering pattern by gender, age, and educational status. All smokers in this study were men; hence, we did not include it in the final model.

\section{Results}

Baseline characteristics of the study participants are presented in Table 1. In all, $43.6 \%$ of the study participants were up to the age of 50 years, and there was a preponderance of females $(57.4 \%)$. The majority of patients (59.4\%) had diabetes for more than five years. Over $78 \%$ of study participants were non smokers, while $9 \%$ were past smokers. About half of the patients reported family history (siblings, parents, and grandparents) of diabetes. In all, $41 \%$ of the diabetic patients were also concurrently diagnosed to have hypertension; however, overall mean systolic and diastolic blood pressure levels of study participants were substantially high. There were also higher mean values of lipid profile (total cholesterol, low-density lipoproteins, and triglycerides) and body mass index. Similarly, the glycemic levels were substantially high among study participants. Over $80 \%$ of the study participants were taking oral hypoglycemic agents, while one-third of the patients were also taking lowdose aspirin on regular basis.
TABLE 1: Background characteristics of study participants.

\begin{tabular}{|c|c|}
\hline Characteristics & $N=887$ \\
\hline \multicolumn{2}{|l|}{ Age (in years) } \\
\hline Up to 50 & $387(43.6)$ \\
\hline More than 50 & $500(56.4)$ \\
\hline \multicolumn{2}{|l|}{ Sex } \\
\hline Females & $509(57.4)$ \\
\hline Males & $368(42.6)$ \\
\hline \multicolumn{2}{|l|}{ Diabetes duration* } \\
\hline 1 to 5 years & $360(40.6)$ \\
\hline More than 5 years & $527(59.4)$ \\
\hline \multicolumn{2}{|l|}{ Status of smoking } \\
\hline Nonsmoker & $694(78.3)$ \\
\hline Past smoker & $80(9.0)$ \\
\hline Current smoker & $113(12.7)$ \\
\hline \multicolumn{2}{|c|}{ Type of treatment for glycemic control } \\
\hline Diet and exercise & $85(9.6)$ \\
\hline Single oral drug & $351(39.5)$ \\
\hline More than one oral drug & $367(41.4)$ \\
\hline Insulin therapy & $84(9.5)$ \\
\hline \multicolumn{2}{|l|}{ Taking low-dose aspirin } \\
\hline Yes & $294(33.1)$ \\
\hline No & $593(66.9)$ \\
\hline \multicolumn{2}{|l|}{ Family history of diabetes } \\
\hline Yes & $420(47.2)$ \\
\hline No & $467(52.8$ \\
\hline \multicolumn{2}{|l|}{ Coexist hypertension } \\
\hline Yes & $363(40.9)$ \\
\hline No & $524(59.1)$ \\
\hline Systolic blood pressure & $134.1 \pm 18.2$ \\
\hline Diastolic blood pressure & $83.9 \pm 11.4$ \\
\hline Total cholesterol & $202.1 \pm 34.1$ \\
\hline Low density lipoproteins & $155.4 \pm 45.1$ \\
\hline Triglycerides & $209.4 \pm 86.1$ \\
\hline Fasting blood glucose & $198.6 \pm 79.7$ \\
\hline Postprandial blood glucose & $256.2 \pm 106.5$ \\
\hline $\mathrm{HbA}_{1} \mathrm{C}^{* *}$ & $7.9 \pm 1.4$ \\
\hline Body mass index & $27.7 \pm 5.1$ \\
\hline
\end{tabular}

Data are presented in means \pm SD or $n(\%)$.

*Duration since diagnosis of diabetes; ${ }^{* *} n=285$.

In Table 2, proportion distribution and clustering pattern of lifestyle factors and their associations with CVD are described. Amongst all, 30.3\% (95\% CI $=27.2-33.2)$ of the diabetic patients had CVD. Over $65 \%$ of participants were physically inactive, $71.4 \%$ had anxiety and/or depression, and $40.1 \%$ were exposed to passive smoking. In the univariate analysis, all the three studied lifestyle factors and their clustering pattern were found to be significantly associated with CVD (physical inactivity: crude OR $=1.7,95 \% \mathrm{CI}=$ 1.2-2.3; psychosocial factors: crude $\mathrm{OR}=2.2,95 \% \mathrm{CI}=1.6-$ 3.2; passive smoking exposure: crude $\mathrm{OR}=1.7,95 \% \mathrm{CI}=$ 1.3-2.3; clustering of any two or three lifestyle factors: crude $\mathrm{OR}=6.1,95 \% \mathrm{CI}=2.7-13.7)$. However, the confounders 
TABLE 2: Proportion distribution and clustering pattern of lifestyle risk factors and their associations with CVD among study participants.

\begin{tabular}{|c|c|c|c|c|}
\hline Characteristics & Overall $n$ & $\begin{array}{c}\text { Having CVD } \\
n(\%)\end{array}$ & $\begin{array}{c}\text { Crude odds ratio } \\
\qquad(95 \% \mathrm{CI})\end{array}$ & $\begin{array}{c}\text { Adjusted odds ratio } \\
(95 \% \mathrm{CI})\end{array}$ \\
\hline \multicolumn{5}{|c|}{ Model I } \\
\hline \multicolumn{5}{|l|}{ Physical activity } \\
\hline Yes & 309 & $72(23.3)$ & 1 & 1 \\
\hline No & 578 & $197(34.1)$ & $1.7(1.2-2.3)$ & $1.6(1.2-2.3)$ \\
\hline \multicolumn{5}{|l|}{ Psychosocial factors } \\
\hline No & 254 & $49(19.3)$ & 1 & 1 \\
\hline Yes & 633 & $220(34.8)$ & $2.2(1.6-3.2)$ & $1.9(1.4-2.8)$ \\
\hline \multicolumn{5}{|l|}{ Passive smoking } \\
\hline No & 531 & $136(25.6)$ & 1 & 1 \\
\hline Yes & 356 & $133(37.4)$ & $1.7(1.3-2.3)$ & $1.7(1.2-2.3)$ \\
\hline \multicolumn{5}{|c|}{ Model II } \\
\hline \multicolumn{5}{|c|}{ Clustering of lifestyle factors } \\
\hline No factor & 78 & $7(9.0)$ & \multicolumn{2}{|c|}{1} \\
\hline 1 factor & 244 & $51(20.9)$ & \multicolumn{2}{|c|}{$2.7(1.2-6.2)$} \\
\hline 2 or three factors & 565 & $211(37.3)$ & \multicolumn{2}{|c|}{$6.1(2.7-13.7)$} \\
\hline
\end{tabular}

After adjustment for age, sex, educational status, and body mass index.

(age, sex, educational status, and body mass index) reported in the literature were not fulfilling the criteria of being a confounding factor in this study except age which was confounded by the exposures of physical inactivity and clustering of lifestyle factors.

In model I of the final multivariable analysis (Table 2), the odds of having CVD were higher among those who were physically inactive (adjusted OR $=1.6 ; 95 \% \mathrm{CI}=1.2-2.3$ ) and had anxiety and/or depression (Adjusted OR $=1.9 ; 95 \%$ $\mathrm{CI}=1.4-2.8)$, and among those exposed to passive smoking (adjusted OR $=1.7 ; 95 \%$ CI $=1.2-2.3$ ) while adjusted for age, sex, educational status, and body mass index. In another model, the odds of having CVD increased with the clustering of lifestyle factors: for having any one factor (adjusted $\mathrm{OR}=2.7 ; 95 \% \mathrm{CI}=1.2-6.2$ ) and for clustering of two or three factors (adjusted OR $=6.1 ; 95 \% \mathrm{CI}=2.7-13.7$ ) after adjusting for the similar factors described in the first model.

The association of sociodemographic characteristics with CVD-related lifestyle factors and their clustering pattern are presented in Table 3. In comparison to males, females were more physically inactive $(57.1 \%$ versus $71.1 \%$; $P<0.001)$. Similarly those with no/less education were more inactive compared to their counterparts having education of more than five years $(77.7 \%$ versus $53.6 \% ; P<0.001)$. Significant majority of study participants having anxiety and/or depression were females $(74.5 \%)$, elderly $(77.0 \%)$, and having no/less education $(75.6 \%)$ compared to their counterparts. Exposure of passive smoking was almost equally distributed among all groups of sociodemographic classes. Clustering of two or three CVD-related lifestyle factors was significantly higher among females compared to males (69.9\% versus $55.3 \% ; P<0.001)$ and elderly compared to younger patients (68.1\% versus $58.0 \% ; P=0.004)$. Clustering of these factors was also higher among study participants having no/less education compared to those with more education $(71.8 \%$ versus $56.2 \% ; P<0.001)$.

\section{Discussion}

It is well known that modifiable lifestyle factors such as physical inactivity, psychosocial stress, and smoking result in an increased risk of CVD, while control of the same substantially decreases the development of CVD $[1,15]$. The results of our study are consistent with those of previous studies and highlight the importance of adequate control of these lifestyle factors, particularly among high-risk people like diabetics, to prevent the development and progression of CVD and its consequences.

In a recently conducted study on healthy Canadian adults [21], only $10 \%$ of the study population was found to be physically inactive, as opposed to $60 \%$ healthy adults in Pakistan [13]. The presence of such high levels of physi-cal inactivity is particularly harmful among people with diabetes, as the presence of diabetes itself confers increased cardiovascular risk, and the coexistence of physical inactivity further amplifies this risk. The high prevalence of physical inactivity in an earlier study from Pakistan [13] and in our study may be attributed to the lack of awareness regarding the role and benefits of exercise in preventing CVD as highlighted from the earlier studies in Pakistan [22]. Furthermore, watching television and playing computer games have considerably risen in this part of the world, along with unavailability of safe playgrounds and walking tracks rendering majority of the population physically inactive [13].

Furthermore, the burden of psychosocial factors which predispose to anxiety and depression is also reported to be more prevalent in developing countries [23]. By using HADS 


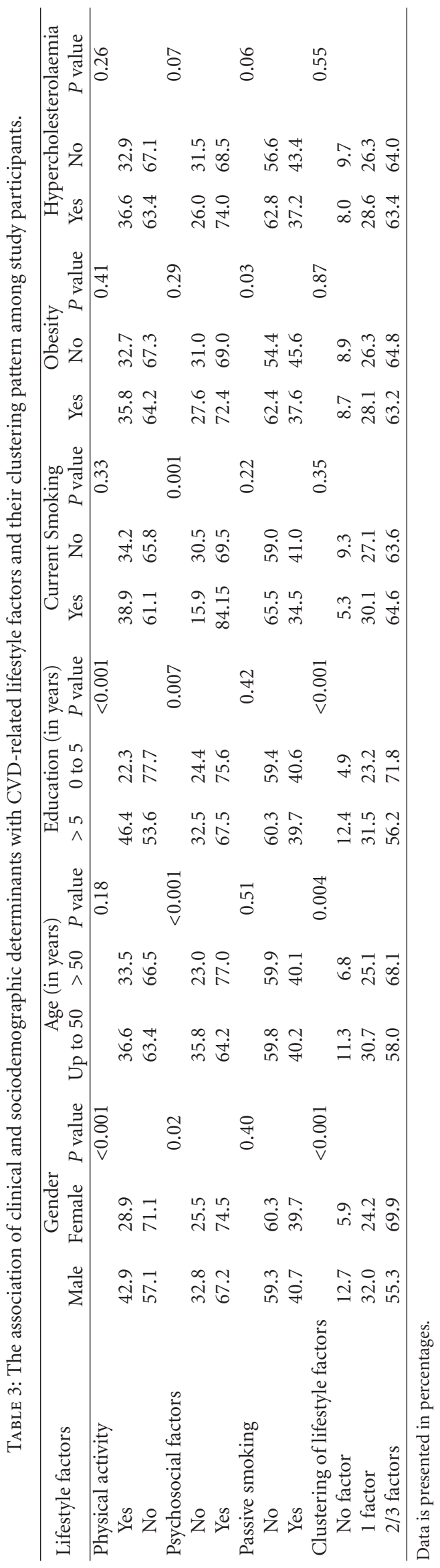


scale, our study showed that more than $70 \%$ of the study participants were suffering from anxiety and/or depression. However, results of a study conducted in the United Kingdom using the same scale revealed substantially lesser proportion of these disorders among diabetic patients [24]. This sharp difference in prevalence of mood disorders may be attributed to the high levels of poverty, poor socioeconomic conditions, and scarce health care facilities and resources in developing countries.

It is evident that smoking is a strong risk factor for developing CVD in general [1] and among diabetics in particular [7]. It is also reported that passive smoking also increases the risk of chronic diseases including CVD $[8,10]$. A community-based survey recently reported that about half of the study participants in Pakistan were exposed to passive smoking [25]. Using the same definition of pas-sive smoking in our study, over one-third of the diabetic population was found to be exposed to passive smoking. This high proportion of exposure reflects the lack of awareness about the hazards of passive smoking among the general population. In Pakistan, in spite of laws and constitutions to restrict smoking at public places, people freely smoke in public transport, shopping malls, restaurants, and even many of the work places and offices. This dreadful scenario exposes higher proportion of people to passive smoking. It is documented that, in Pakistan, up to $34 \%$ adults smoke cigarettes [25]. However, in our study, $13 \%$ of the study participants were reported as being current smokers and all of them were males. This lower prevalence can be attributed to the fact that in this study, interviews were conducted in the presence of patients' family members/attendants, raising the possibility that majority of the participants did not admit that they smoked, as it is viewed as a socially unacceptable habit in this part of the world. Another possible explanation for the lower prevalence of smoking may be due to the fact that patients might have given up smoking after developing diabetes and its complication. Furthermore, previous work reported was done only in men [25], and it is well reported that when compared to women, smoking among men is more prevalent in both developed as well as in developing countries $[13,26]$.

Recently, data collected from a multisite five Asian countries survey [27] revealed that a substantial proportion of study population had clustering of lifestyle risk factors, and similar findings were also reported in a community-based study from urban Pakistan [13]. In our study, a very small proportion of the participants were found to have none of CVD-related lifestyle factors, while one-fourth had one of these risks, and majority had clustering of two or three factors. This pattern of unhealthy lifestyle clustering reflects the possibility of poor awareness and harmful attitude and practices regarding healthy behaviors. Results of this study also endorse the existing literature that physical inactivity, adverse psychosocial factors, and exposure to smoking are strongly associated with CVD $[1,15]$, and clustering of these factors enhances the risk further $[13,28]$.

With the increase in prevalence of CVD-related modifiable lifestyle factors among high-risk population like diabetics in developing countries, identification of specific tar- get groups within such population is important in order to introduce comprehensive and integrated preventive strategies aimed to reduce CVD. The presence of cardiovascular risk factors in both the genders has been studied extensively. Though literature from the Western world has identified females to have a lower risk of developing CVD [29], evidence from South Asia reveals that both men and women bear an equal risk of developing these diseases [30]. Furthermore, a study from Pakistan reports women to have a greater risk of developing CVD than men [31]. The results of our study reveal that all the CVD-related lifestyle factors and their clustering are significantly higher among women. Hence, the female population in our study was found to be at higher risk of developing CVD owing to increased prevalence of unhealthy lifestyle behaviors. The presence of increased CVD-related risk factors in women can be attributed to the fact that up to $50 \%$ males have been reported to smoke at home [25], thus exposing the females to passive smoking as well. Furthermore, the social status of women in our patriarchal culture leads to increased prevalence of psychosocial risk factors among them. Moreover, cultural restrictions limit females to going outside their homes with-out an accompanying male family member resulting in decreased likelihood for joining sport physical and exercise centers and gymnasiums.

It is well known that increasing age is associated with increased cardiovascular risk due to acceleration of atherosclerosis [32] and other cardiovascular risk factors [16]. This study showed that up to $80 \%$ of the older population was found to have anxiety and/or depression. This increased psychosocial suffering may be attributed to the presence of more chronic illnesses and CVD as well as challenging life situations such as being more isolated and dependent. Clustering of two or more lifestyle risk factors was also found to be present in up to $70 \%$ of the elderly patients, providing further explanation for increased cardiovascular risk in this age group. These results are in accordance with results of various studies conducted elsewhere $[16,27]$.

It has been reported that higher level of education has a protective role with regard to healthy lifestyle and behaviors $[33,34]$ as it leads to greater awareness and improved conscience regarding health. The results of our study also suggest low prevalence of CVD-related factors such as physical inactivity and anxiety and/or depression among people having more years of education. Similarly, clustering of these risk factors was in lesser proportion among the educated participants, reinforcing the fact that people with less education have an increased risk of unhealthy lifestyle practices.

The overall metabolic control (blood pressure levels, glycemic control, lipid levels, and body mass index) of our study participants was poor. It is well reported that the management and control of diabetes is a real challenge for health care providers as well as people having diabetes $[7,22]$. Many possible reasons and explanations exist for poor control of disease, like lack of knowledge about the disease among diabetic patients [35], poor quality of care 
provided by treating physicians [22], and excessive cost for the management of diabetes $[6,36]$ which many patients cannot afford in resource-constrained countries.

This study also has certain limitations. Since this study employs a cross-sectional study design, temporal relation between CVD and its lifestyle factors could not be identified. Coronary artery disease is often silent with regards to symptoms in diabetic subjects, and therefore noninvasive studies (such as myocardial scintigraphy and dobutamine stress echocardiography) should have been performed for a thorough evaluation of the cardiac status. However, due to cost and resource limits, we have not evaluated the cardiac status using these tests. Similarly, $\mathrm{HbA}_{1} \mathrm{C}$, another comparatively costly test, is recommended to evaluate the overall glycemic control, but it was only reported in 285 patients. Therefore, we cannot comment about the overall glycemic control of study participants during the period of time. All the smokers in this study were male; hence, this important factor was not included in the final analysis. Possibility of recall bias exists regarding some information stated by patients. We did not inquire about the dietary habits of study participants which is an imperative lifestyle factor for CVD. All the study centers were from one metropolitan city; therefore, it may not be possible to generalize the findings to the rural population that might have different behaviors. Furthermore, being a cross-sectional study, we cannot assess the impact of successful medical treatment on the occurrence of CVD. Age, sex, educational status, and body mass index are well known confounders for lifestyle factors with CVD $[7,13,37]$; however, in this study except for the age, other established confounders were not found to be associated with CVD, lifestyle risk factors, and their clustering pattern.

\section{Conclusion}

This multicenter study highlights very high prevalence and clustering of CVD-related lifestyle factors, particularly physical inactivity, anxiety, depression, and exposure to passive smoking. The presence of such a high burden of these factors among diabetic patients is a cause of concern and requires urgent interventions in order to prevent and control CVD. These interventions should be based on a comprehensive and integrated approach covering all of these lifestyle factors rather than any single factor to anticipate their cumulative effects. We recommend that health care providers should provide awareness and education regarding CVD risk factors and their prevention to patients and their families/caregivers. Furthermore, safe walking tracks, playgrounds, and relaxation avenues should also be made available to allow more people to engage in physical activities and relaxation programs. Laws should be strictly imposed to prohibit smoking at public places. Women should be empowered and be allowed to follow a healthy lifestyle. Further research is suggested to explore this very important avenue in more detail and to design and test interventions accordingly.

\section{Conflict of Interests}

The authors declare that there is no conflict of interests.

\section{Acknowledgments}

The authors are thankful to the management and staff of the studied clinics for giving permission to conduct this study and for their support throughout. They are grateful to all the patients who participated in this study. They acknowledge Drs. Hafsa Raheel, Waqar Khusk, Asad Afridi, Nadya Khuwaja, and Wahid Bux Soomro for conducting all patients' interviews and reviewing patient's medical files. They are also thankful to Dr. Niloufer Sultan Ali (Associate Professor) and Ms. Kashmira Nanji (Senior InstructorResearch) Department of Family Medicine, Aga Khan University, Pakistan for their valuable conceptual review and comments on the paper. They acknowledge Dr. Zafar Fatmi (Associate Professor and Director Residency Program) and Dr. Farzana Nawaz Ali (Intern-Research) for reviewing the paper for any possible typographical and grammatical error.

\section{References}

[1] World Health Organization, "Cardiovascular disease Fact sheet $N^{\circ} 317$," http://www.who.int/mediacentre/factsheets/ fs317/en/index.html.

[2] S. Wild, G. Roglic, A. Green, R. Sicree, and H. King, "Global prevalence of diabetes: estimates for the year 2000 and projections for 2030," Diabetes Care, vol. 27, no. 5, pp. 10471053, 2004.

[3] A. S. Shera, F. Jawad, and A. Maqsood, "Prevalence of diabetes in Pakistan," Diabetes Research and Clinical Practice, vol. 76, no. 2, pp. 219-222, 2007.

[4] A. Schainberg, A. Ribeiro-Oliveira, and J. M. Ribeiro, "Is there a link between glucose levels and heart failure? An update," Arquivos Brasileiros de Endocrinologia e Metabologia, vol. 54, no. 5, pp. 488-497, 2010.

[5] Z. Milicevic, I. Raz, S. D. Beattie et al., "Natural history of cardiovascular disease in patients with diabetes: role of hyperglycemia," Diabetes Care, vol. 31, 2, pp. 155-160, 2008.

[6] L. A. Khowaja, A. K. Khuwaja, and P. Cosgrove, "Cost of diabetes care in out-patient clinics of Karachi, Pakistan," BMC Health Services Research, vol. 7, article 189, 2007.

[7] A. K. Khuwaja, G. Rafique, F. White, and S. I. Azam, "Macrovascular complications and their associated Factors among persons with Type 2 diabetes in Karachi, Pakistana multi-center study," Journal of the Pakistan Medical Association, vol. 54, no. 2, pp. 60-66, 2004.

[8] G. S. Metsios, A. D. Flouris, M. Angioi, and Y. Koutedakis, "Passive smoking and the development of cardiovascular disease in children: a systematic review," Cardiology Research and Practice, vol. 2011, Article ID 587650, 6 pages, 2011.

[9] P. Tanuseputro, D. G. Manuel, M. Leung, K. Nguyen, H. Johansen, and Canadian Cardiovascular Outcome Research Team, "Risk factors for cardiovascular disease in Canada," Canadian Journal of Cardiology, vol. 19, no. 11, pp. 1249-1259, 2003.

[10] W. Wen, X. O. Shu, Y. T. Gao et al., "Environmental tobacco smoke and mortality in Chinese women who have never smoked: prospective cohort study," British Medical Journal, vol. 333, no. 7564, pp. 376-379, 2006. 
[11] M. Kivimäki, M. Virtanen, M. Elovainio, A. Kouvonen, A. Vää nänen, and J. Vahtera, "Work stress in the etiology of coronary heart disease-a meta-analysis," Scandinavian Journal of Work, Environment and Health, vol. 32, no. 6, pp. 431-442, 2006.

[12] World Health Organization, "Global strategy on diet, physical activity and health. Diet and physical activity: a public health priority," http://www.who.int/dietphysicalactivity/.

[13] A. K. Khuwaja and M. M. Kadir, "Gender differences and clustering pattern of behavioural risk factors for chronic non-communicable diseases: community-based study from a developing country," Chronic lllness, vol. 6, no. 3, pp. 163-170, 2010.

[14] A. K. Khuwaja, R. Qureshi, and Z. Fatmi, "Noncommunicable diseases and injuries: action needed in South Asia too," PLoS Medicine, vol. 4, no. 1, pp. 192-193, 2007.

[15] World Health Organization, "World Health Report 2002. Reducing risk, promoting healthy life," Tech. Rep., World Health Organization, Geneva, Switzerland.

[16] L. Zhang, L. Q. Qin, H. Y. Cui, A. P. Liu, and P. Y. Wang, "Prevalence of cardiovascular risk factors clustering among suburban residents in Beijing, China," International Journal of Cardiology, vol. 151, no. 1, pp. 46-49, 2011.

[17] Y. Ohta, T. Tsuchihashi, U. Onaka, and E. Hasegawa, "Clustering of cardiovascular risk factors and blood pressure control status in hypertensive patients," Internal Medicine, vol. 49, no. 15, pp. 1483-1487, 2010.

[18] "International Physical Activity Questionnaire (IPAQ)," http://www.ipaq.ki.se/.

[19] A. S. Zigmond and R. P. Snaith, "The hospital anxiety and depression scale," Acta Psychiatrica Scandinavica, vol. 67, no. 6, pp. 361-370, 1983.

[20] D. B. Mumford, I. A. K. Tareen, M. A. Z. Bajwa, M. R. Bhatti, and R. Karim, "The translation and evaluation of an Urdu version of the hospital anxiety and depression scale," Acta Psychiatrica Scandinavica, vol. 83, no. 2, pp. 81-85, 1991.

[21] S. N. Bryan and P. T. Katzmarzyk, "Are Canadians meeting the guidelines for moderate and vigorous leisure-time physical activity?" Applied Physiology, Nutrition and Metabolism, vol. 34, no. 4, pp. 707-715, 2009.

[22] I. S. Azam, A. K. Khuwaja, G. Rafique, and F. White, "Assessment of quality of care for the management of type 2 diabetes: a multicentre study from a developing country," Quality in Primary Care, vol. 18, no. 3, pp. 207-214, 2010.

[23] A. K. Khuwaja, R. Qureshi, and S. I. Azam, "Prevalence and factors associated with anxiety and depression among family practitioners in Pakistan," Journal of the Pakistan Medical Association, vol. 54, no. 2, pp. 45-49, 2004.

[24] M. M. Collins, P. Corcoran, and I. J. Perry, "Anxiety and depression symptoms in patients with diabetes," Diabetic Medicine, vol. 26, no. 2, pp. 153-161, 2009.

[25] A. K. Khuwaja and M. M. Kadir, "Smoking among adult males in an urban community of Karachi, Pakistan," Southeast Asian Journal of Tropical Medicine and Public Health, vol. 35, no. 4, pp. 999-1004, 2004.

[26] N. S. Parikh, M. C. Fahs, D. Shelley, and R. Yerneni, "Health behaviors of older Chinese adults living in New York City," Journal of Community Health, vol. 34, no. 1, pp. 6-15, 2009.

[27] S. M. Ahmed, A. Hadi, A. Razzaque et al., "Clustering of chronic non-communicable disease risk factors among selected Asian populations: levels and determinants," Glob Health Action, vol. 2, 2009.

[28] N. K. Vikram, N. Tandon, A. Misra et al., "Correlates of type 2 diabetes mellitus in children, adolescents and young adults in north India: a multisite collaborative case-control study," Diabetic Medicine, vol. 23, no. 3, pp. 293-298, 2006.

[29] A. Alkerwi, N. Sauvageot, A. F. Donneau et al., "First nationwide survey on cardiovascular risk factors in Grand-Duchy of Luxembourg," BMC Public Health, vol. 10, article 468, 2010.

[30] T. H. Jafar, F. H. Jafary, S. Jessani, and N. Chaturvedi, "Heart disease epidemic in Pakistan: women and men at equal risk," American Heart Journal, vol. 150, no. 2, pp. 221-226, 2005.

[31] T. H. Jafar, "Women in Pakistan have a greater burden of clinical cardiovascular risk factors than men," International Journal of Cardiology, vol. 106, no. 3, pp. 348-354, 2006.

[32] G. A. Heckman and R. S. McKelvie, "Cardiovascular aging and exercise in healthy older adults," Clinical Journal of Sport Medicine, vol. 18, no. 6, pp. 479-485, 2008.

[33] E. Peters, D. P. Baker, N. F. Dieckmann, J. Leon, and J. Collins, "Explaining the effect of education on health: a field study in Ghana," Psychological Science, vol. 21, no. 10, pp. 1369-1376, 2010.

[34] M. Z. Maksimović, H. D. Vlajinac, D. J. Radak, J. M. Maksimović, J. M. Marinković, and J. B. Jorga, "Association of socioeconomic status measured by education and risk factors for carotid atherosclerosis: cross-sectional study," Croatian Medical Journal, vol. 49, no. 6, pp. 824-831, 2008.

[35] G. Rafique, S. I. Azam, and F. White, "Diabetes knowledge, beliefs and practices among people with diabetes attending a university hospital in Karachi, Pakistan," Eastern Mediterranean Health Journal, vol. 12, no. 5, pp. 590-598, 2006.

[36] A. K. Khuwaja, L. A. Khowaja, and P. Cosgrove, "The economic costs of diabetes in developing countries: some concerns and recommendations," Diabetologia, vol. 53, no. 2, pp. 389-390, 2010.

[37] A. K. Khuwaja, S. Lalani, R. Dhanani, I. S. Azam, G. Rafique, and F. White, "Anxiety and depression among outpatients with type 2 diabetes: a multi-centre study of prevalence and associated factors," Diabetology \& Metabolic Syndrome, vol. 2, p. 72, 2010. 


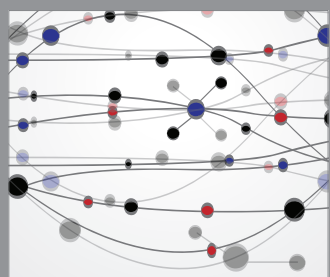

The Scientific World Journal
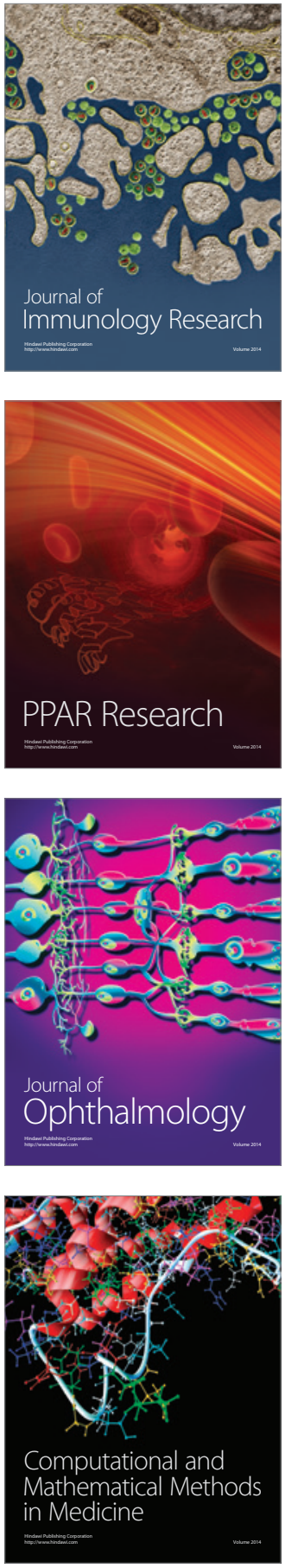

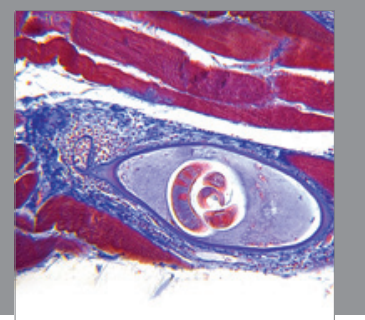

Gastroenterology

Research and Practice
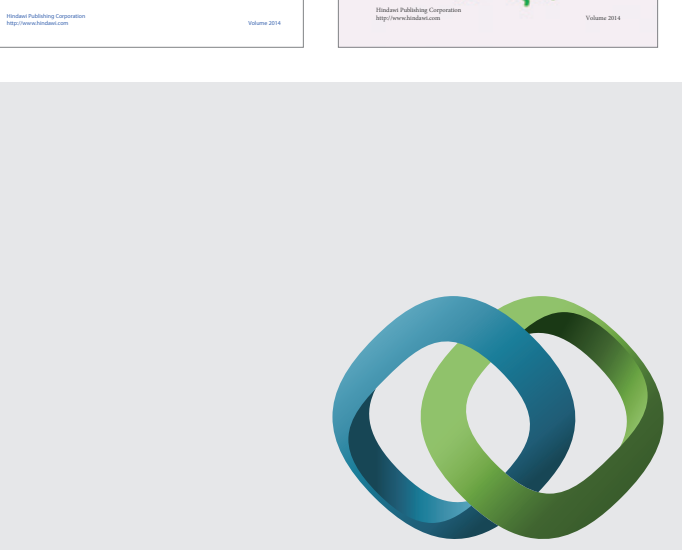

\section{Hindawi}

Submit your manuscripts at

http://www.hindawi.com
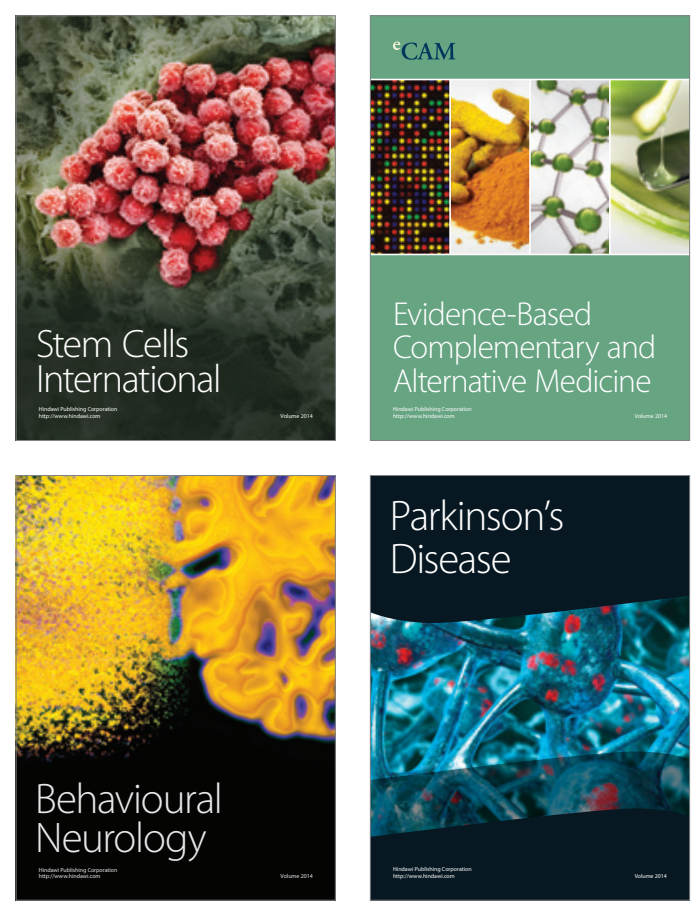

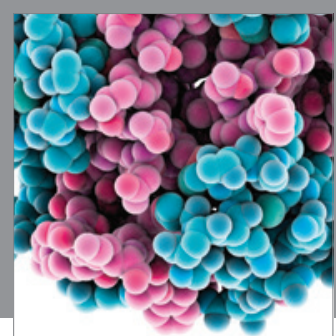

Journal of
Diabetes Research

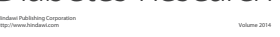

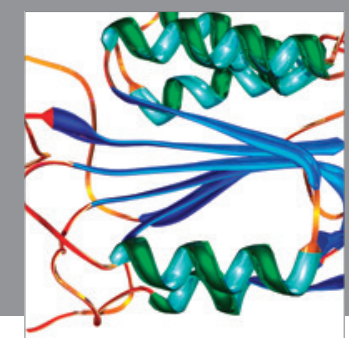

Disease Markers
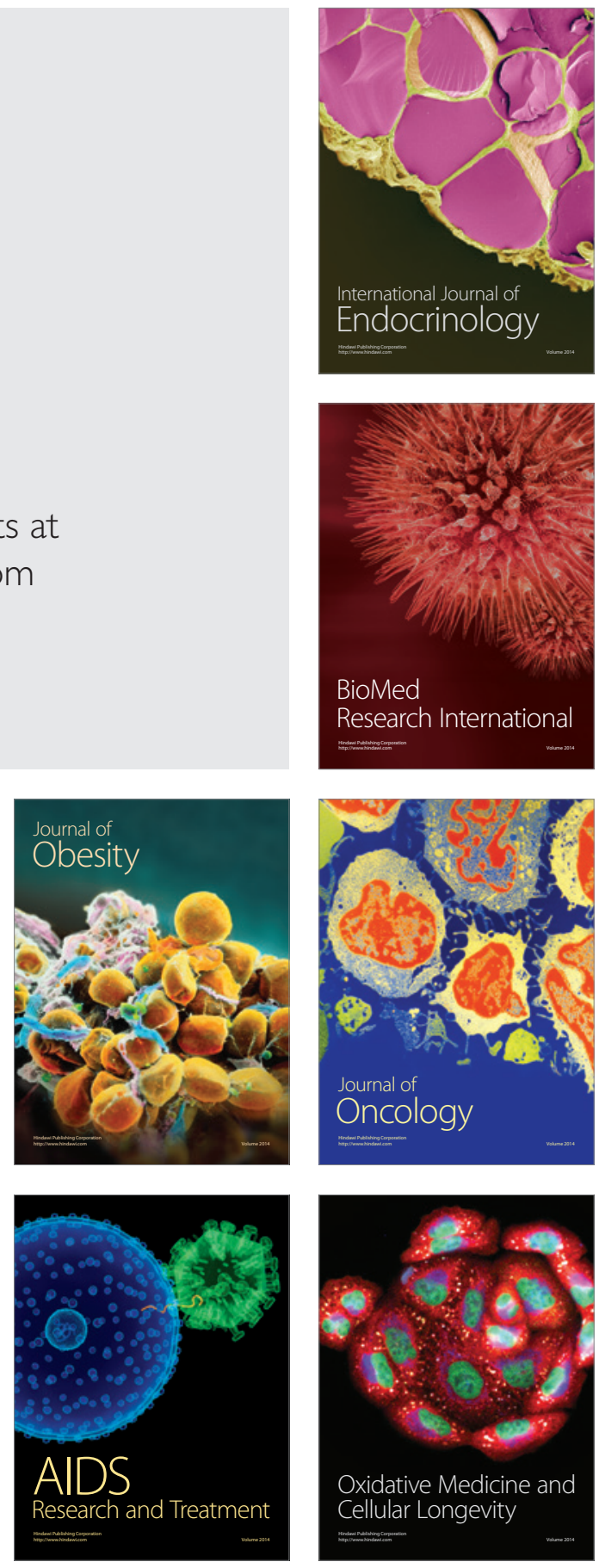